\title{
Compact Grid Representation of Graphs ${ }^{\star}$
}

\author{
José Cáceres $^{1}$, Carmen Cortés ${ }^{2}$, Clara Isabel Grima ${ }^{2}$, Masahiro Hachimori ${ }^{3}$, \\ Alberto Márquez ${ }^{2}$, Raiji Mukae ${ }^{4}$, Atsuhiro Nakamoto ${ }^{4}$, Seiya Negami ${ }^{4}$, \\ Rafael Robles ${ }^{2}$, and Jesús Valenzuela ${ }^{2}$ \\ 1 Universidad de Almería, Spain \\ jcaceres@ual.es \\ 2 Universidad de Sevilla, Spain \\ \{ccortes, grima, almar, rafarob, jesusv\}@us.es \\ 3 Tsukuba University, Japan \\ hachi@sk.tsukuba.ac.jp \\ 4 Yokohama National University, Japan \\ \{mkerij,nakamoto, negamij\}@edhs.ynu.ac.jp
}

\begin{abstract}
A graph $G$ is said to be grid locatable if it admits a representation such that vertices are mapped to grid points and edges to line segments that avoid grid points but the extremes. Additionally $G$ is said to be properly embeddable in the grid if it is grid locatable and the segments representing edges do not cross each other. We study the area needed to obtain those representations for some graph families.
\end{abstract}

Keywords: graph drawing, grid locatable, grid embeddable, chromatic number.

\section{Introduction}

Graph drawing applies topology and geometry to derive suitable representations of graphs. Particularly, grid representations of graphs have attracted the attention of many researchers (see, for example 4711]).

A grid point is a point of the plane having integer coordinates. A line segment $s$ joining two grid points is said to be primitive if the only grid points in $s$ are its extremes.

A graph $G$ is said to be grid locatable (or locatable in the grid), if each vertex is represented by a grid point and each edge by a primitive segment joining its extremes. $G$ is said to be properly embeddable in the grid ( $p$-embeddable for short) if it is grid locatable and the segments representing edges do not cross each other. Observe, that the graphs so obtained are subgraphs of the visibility graph of the grid points, where two points are visible if there is no other grid point between them (this visibility graph was defined, for example, in [12).

\footnotetext{
* Partially supported by projects JA-FQM305, ESF EUROCORES programme EuroGIGA - ComPoSe IP04 - MICINN project EUI-EURC-2011-4306, MTM200805866-C03-01, and PAI-FQM141.
} 
In this way, a graph is grid locatable (resp. p-embeddable) if it is isomorphic to a subgraph (resp. plane subgraph) of the visibility graph of the grid points.

The following characterization is shown in 9 .

Theorem 1. [9] A graph $G$ is grid locatable if and only if $G$ is 4-colorable.

One of the reasons to study grid representations of graphs is to test how compact a given representation can be. Thus, a very important question in this kind of representation is to minimize the area needed to represent a given graph.

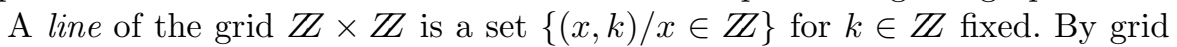
locating (or p-embedding) a graph $G$ in $\ell$ lines we mean to locate (or embed) $G$ in $Z \mathbb{Z} \times \mathbb{Z}$ mapping the vertices to grid points in $\ell$ consecutive lines (there is no unused line between two lines in our drawing).

In [9], an upper bound on the number of lines needed to grid locate a graph in the grid is given.

Corollary 1. [9] A graph $G$ with chromatic number $\chi(G) \leq 4$ can be located in the grid in, at most, $\chi(G)$ lines.

Although the authors give an upper bound to the number of lines needed in order to grid locate a graph with $\chi(G) \leq 4$, in general, that upper bound is not tight. In this work, we deal with this problem. Namely, to find a tighter upper bound of the area (number of lines) needed to grid locate or p-embed a given graph in the grid.

A simple but useful observation is the following:

Remark 1. An edge joining two grid points $\left(x_{1}, y_{1}\right)$ and $\left(x_{2}, y_{2}\right)$ is primitive if and only if $\left|x_{1}-x_{2}\right|$ and $\left|y_{1}-y_{2}\right|$ are relatively prime to each other.

The structure of this paper is as follows. In Sect. 2, we show some results about p-embeddability of graphs in the grid. In Sect. 3, we provide our main results, giving a characterization of those graphs that are grid locatable in two and three lines. We shall conclude with some remarks and open problems.

\section{P-Embedding a Graph in the Grid}

Until very recently, it was not known whether any planar graph could be pembedded in the plane or not, and there were only some partial results on the subject (see 9]). This problem has just been solved in the affirmative independently by M. Balko [1] and Flores-Peñaloza et al. [8], although the grid size of the resulting representation is still excessively large. So it makes sense to find bounds on the size of the grid for some specific families of graphs, as we do below for bipartite graphs.

Proposition 1. Any plane bipartite graph can be p-embedded in the grid. 
Proof. In 2 it is shown how to embed a quadrangulation in the grid. They prove firstly that any planar quadrangulation admits two different representations in two 2-books (in a 2-book representation, vertices are mapped to a line, called the spine, and edges are represented by crossing-free curves which do not cross the spine either). Then, they assign to each vertex the coordinates of its relative positions in the spines of both 2-books. A similar representation can be performed by forcing the differences of the $x$-coordinates to be relatively prime each other, guaranteeing the condition of primitiveness. This can be achieved by replacing the first $n$-positive integer $x$-coordinates of the graph by the first $n$ primes.

As a consequence of the proof of Proposition 1 and applying a direct implication of the well known Prime Number Theorem that establishes that the asymptotic expression for the $n$th prime number is $n \log n$ (see [10]), we obtain a (not really tight) upper bound on the area needed to p-embed a bipartite graph in the grid.

Corollary 2. Any plane bipartite graph with $n$ vertices can be $p$-embedded in a rectangle of area $O\left(n^{2} \log n\right)$.

On the other hand, a complete characterization is given in [5] for those graphs that are p-embedabble in two lines.

Proposition 2. [5] A graph $G$ is p-embeddable in two lines if and only if $G$ can be extended to a maximal outerplanar graph such that its dual (excluding the vertex representing the outerface) is a path.

It is known that if a graph is grid locatable in the plane, then it is grid locatable in at most four lines, however, it is hopeless to try to obtain a similar result regarding p-embeddability. This is because some graphs with $n$ vertices, as the nested triangles graphs (see Fig. 1), are shown to require a grid of size $(2 n / 3-1) \times$ $(2 n / 3-1)$ for a plane straight-line drawing $[6$. Then, it seems to be worthwhile to improve the bounds on the area needed for certain families of graphs to be $\mathrm{p}-$-embeddable.

\section{Locating a Graph in the Grid}

As we said above, the upper bound provided in the number of lines required to grid locate a graph given in Theorem 1 is not always optimal. In this section we tackle the problem of minimizing such number of lines. In other words, given a concrete 4-colorable graph, how many lines are needed to represent it? Next result answers this question for outerplanar graphs.

Proposition 3. Any outerplanar graph can be located in two lines.

Proof. We will show that any maximal outerplanar graph $G$ can be located in two lines (and then the result for any outerplanar graph is straightforward). 


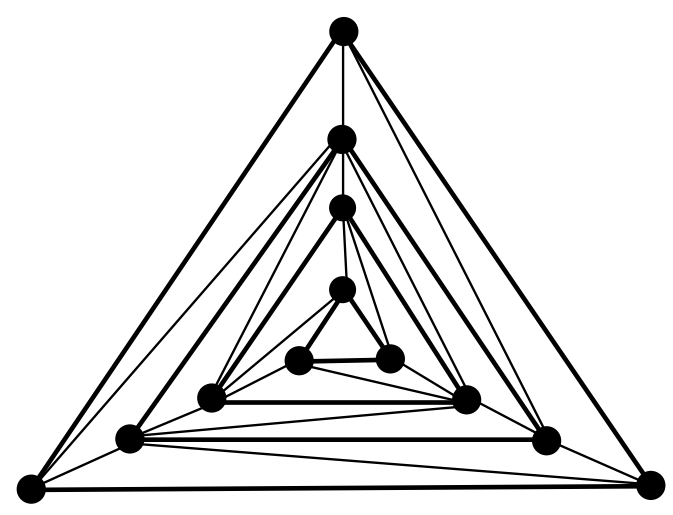

Fig. 1. The nested triangle

The proof can be obtained inductively, just starting by a triangle of the maximal outerplanar graph $G$ and adding its adjacent triangles (at most three) taking into account some easy rules.

Assume that a subgraph $G^{\prime}$ (made of adjacent triangles) of $G$ has already been located in two lines and that a new triangle $u v w$ of $G$ is going to be added and it shares the edge $u v$ with a previously located triangle of the subgraph $G^{\prime}$. Then, one of the following situations holds:

- Either $u$ and $v$ are mapped to contiguous vertices in the same line, or

$-u$ and $v$ are mapped to vertices in different lines but at least one of them is not adjacent (in $G^{\prime}$ ) to one of its contiguous vertices in the same line.

In the fist situation, we add the new vertex to the other line and in the second case, we add the new vertex to the place of the contiguous vertex that is not adjacent. In this last case, we possibly have to move some of the previous vertices in one of the lines (the one the new vertex is added to) in order to make room for the new vertex $w$. Figure 2 shows how to add a vertex in this last case. Triangle 129 is going to be added and it shares the edge 12 with the previously located triangle 123. Since 1 and 4 are not adjacent, then vertices 4 and 8 move to map the vertex $w=9$ (the tiny triangles in the central image mean that one of those points is in the outerface).

We can describe the general structure of any graph that is grid locatable in two or three lines.

Theorem 2. Let $G=(V, E)$ be a graph such that $\chi(G) \leq 4$. Then,

1. $G$ is grid locatable in two lines if and only if $V$ can be partitioned into two subsets $V_{1}, V_{2}$, such that the subgraph of $G$ induced by $V_{i}, i=1,2$, is a disjoint union of paths. 

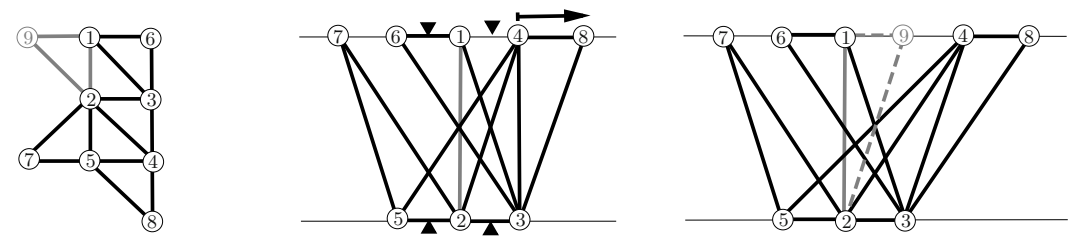

Fig. 2. Adding a vertex in the second case

2. $G$ is grid locatable in three lines if and only if $V$ can be partitioned into three subsets $V_{1}, V_{2}, V_{3}$, such that the subgraph of $G$ induced by $V_{1}$ is a disjoint union of paths, and $V_{2}$ and $V_{3}$ are independent sets.

Proof. The proof of (1) is trivial.

To prove (2), assume first that $G$ is grid locatable in three lines and that those lines are numbered 1, 2 and 3 from bottom to top. We assign all the vertices in line 2 to $V_{1}$, vertices with odd abscissae in lines 1 and 3 to $V_{2}$ and vertices with even abscissae in lines 1 and 3 to $V_{3}$ (see Fig. [3).

To prove the other implication, once the set $V$ is partitioned into sets $V_{1}, V_{2}, V_{3}$, we can draw the graph in three lines numbered 1, 2 and 3 from bottom to top as follows. Since the subgraph of $G$ induced by $V_{1}$ is a disjoint union of paths, we embed those paths in line 2. Then, vertices of $V_{2}$ are mapped to vertices with odd abscissae of line 1 , and vertices in $V_{2}$ are mapped to vertices with even abscissae in line 3.

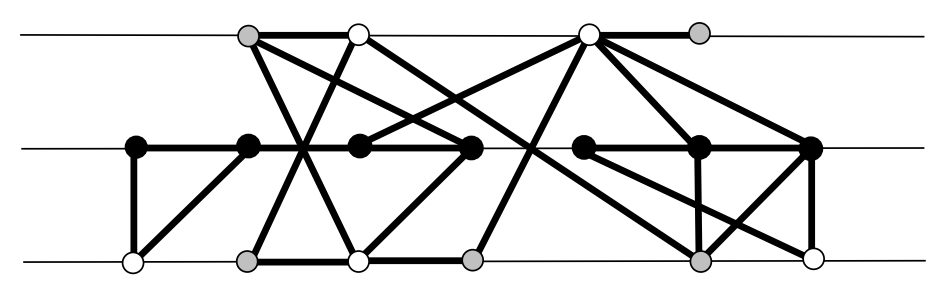

Fig. 3. The coloring of the vertices of the graph located in three lines

Theorem 2 can be used to obtain examples of planar graphs which prove that the bound given in Corollary 1 is sometimes reached. For instance, consider the graph depicted in Fig. 4, it has chromatic number 4 and it requires exactly four lines to be located in the grid. This is because (up to permutation of the colors) there is only one 4-coloring and there does not exist any assignment of the colors avoiding vertices of degree 3 in the subgraph induced by two colors. 


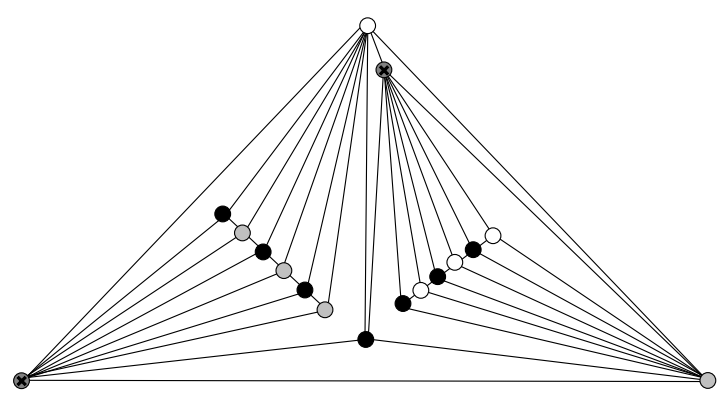

Fig. 4. Four lines are needed to locate this graph in the grid

Additionally, Theorem 2 is one of the main tools used to prove some results regarding grid locatability when the maximum degree of the graph is bounded.

From now on, for a graph $G, \delta(v)$ denotes the degree of a vertex $v$ and $\Delta(G)$ the maximum vertex degree in $G$. Similarly, for a directed graph $G, \delta^{-}(v)$ denotes the in-degree of a vertex $v$ and $\Delta^{-}(G)$ the maximum vertex in-degree in $\mathrm{G}$.

Lemma 1. Let $G$ be a connected, directed, acyclic and not isomorphic to $K_{5}$ graph, with $\Delta(G) \leq 4$ and $\Delta^{-}(G) \leq 3$. Then $G$ is grid locatable in two lines.

Proof. We are going to make use of the first statement of Theorem 2 by partitioning the vertices of $G$ into two subsets, $V_{1}$ and $V_{2}$, such that the subgraphs of $G$ induced by them are disjoints union of paths.

Each directed acyclic graph induces a partial order $\leq$ on its vertices, where $u \leq v$ if there exists a directed path from $u$ to $v$. Once the vertices are sorted, we sequentially assign them labels 1 or 2 as follows: Vertex with no incoming edges are marked with label 1, and the rest are assigned the less popular label among those used by their tails (for vertices with exactly two incoming edges with different colors in their tails, we choose any of them).

After this procedure, the subgraphs of $G$ induced by each label class, $\left.G\right|_{V_{1}}$ and $\left.G\right|_{V_{2}}$, may have vertices of degree three but this may only occur when a vertex $v$ is the tail of two or three vertices of its same color class, as it is depicted in Fig. 5 . This is avoided by sweeping the vertices of $G$ in reverse order and changing the labels of such vertices. Note that to change the label of a vertex may produce a new degree three vertex, but always in a vertex that has not been visited yet.

It only rests to prove that the graphs $\left.G\right|_{V_{1}}$ and $\left.G\right|_{V_{2}}$, regardless of the edges orientation, have no cycles.

First note that a vertex that have changed its label in the previous sweeping cannot take part of a cycle, since it becomes a vertex with degree one in its class. This is obvious in the right case in Fig. 5. In the left case, $v$ could be a vertex of degree two in $\left.G\right|_{V_{2}}$ if its tail with label 1 would change to label 2 in a later step of the sweeping. But this can not happen since now its head, $v$, has a different label than it. 

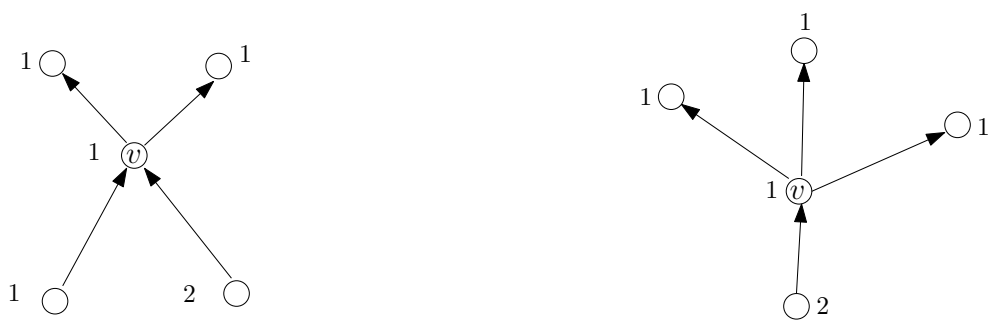

Fig. 5. Vertices with degree 3 in the subgraph induced by one of the color classes

Finally, if there is a monochrome cycle, there must be at least a vertex $v$ with two incoming edges (since $G$ is a directed acyclic graph). But if two incoming edges have the same color in their tails then $v$ should have been previously assigned a different color and we have already shown that no vertex of the cycle has changed its color, what is a contradiction.

Theorem 3. Let $G$ be a connected graph, not isomorphic to $K_{5}$, with $\Delta(G) \leq 4$.

a) If $G$ is 4-regular, then $G$ is grid locatable in three lines.

b) If there exists a vertex $v$ such that $\delta(v) \leq 3$, then $G$ is grid locatable in two lines.

\section{Proof. Proof of a):}

Given that $\Delta(G) \leq 4$, since $G$ is 4-regular, every vertex has -at least- two non-adjacent neighbors. Let $x_{n}$ be one of its vertices, with two non-adjacent neighbors $x_{1}$ and $x_{2}$.

By mimicking Brooks' greedy algorithm to color a graph [3], we assign colors $\{1,2,3,4\}$ to the vertices as we describe below.

Sort the vertices of $G$ so that if $i<j$ then $d\left(x_{n}, x_{i}\right) \geq d\left(x_{n}, x_{j}\right)$ for $i \geq 3$ (for vertices at the same distance from $x_{n}$, we freely choose how to sort them) and sequentially color them as follows:

1. Assign color 1 both to $x_{1}$ and $x_{2}$.

2. For $i \geq 3$, assign preferentially colors 1 or 2 to $x_{i}$ unless colors 3 or 4 are bound to be used.

First, we show that this assignment of colors provides a 4-coloring of $G$. This can be done by induction: Obviously, it is a proper coloring for $x_{1}$ and $x_{2}$ since they are not adjacent. It also works for $x_{n}$ since both $x_{1}$ and $x_{2}$ has the same color. Now assume that, for $3 \leq i<n, x_{i}$ has already been well-colored and let us color $x_{i+1}$. There must exist a vertex $x_{k}, i+1<k \leq n$ such that $d\left(x_{n}, x_{k}\right) \leq$ $d\left(x_{n}, x_{i+1}\right)$. Since $\delta\left(x_{k}\right) \leq 4$, at most three of its neighbors have been already colored and hence there is still a free color for $x_{i+1}$.

Next we prove that the subgraph induced by colors 3 and 4 , denoted $H_{34}$, is a disjoint union of paths. 
Observe that $\delta_{H_{34}}\left(x_{i}\right) \leq 2$ for every $x_{i} \in V\left(H_{34}\right), i \neq n$. On the other hand, if $x_{n} \in V\left(H_{34}\right)$ then $\delta_{H_{34}}\left(x_{n}\right)=1$. This proves that $H_{34}$ is the union of paths and cycles and, in the case of having cycles, $x_{n}$ does not take part of none of them.

If $H_{34}$ is supposed to include a cycle, consider the vertex $x_{j}$ whose label $j(<n)$ is the maximum among the vertices lying on the cycle. Since $x_{j}$ has either color 1 or 2 and colors 3 and 4 are assigned preferentially, $x_{j}$ has (at least) two neighbors previously colored 3 and 4 and still another neighbor to be colored later, what implies $\delta\left(x_{j}\right) \geq 5$, a contradiction.

The proof is concluded by virtue of Theorem 2 .

Proof of $\mathbf{b})$ : Assume now the case of $G$ having a vertex, say $x_{n}$, with degree at most three.

We sort the vertices of $G$ so that if $i<j$ then $d\left(x_{n}, x_{i}\right) \geq d\left(x_{n}, x_{j}\right)$ for $1 \leq i<n$ (again, for vertices at the same distance from $x_{n}$, we freely choose how to sort them).

This ordering induces an acyclic digraph in $G$ with maximum in-degree 3 . Now, applying Lemma 1 the result holds.

\section{Concluding Remarks and Open Questions}

As it was said in the introduction, grid embeddings of graphs as subgraphs of the visibility graph of the grid points has been considered previously in the literature, mainly in 9 .

But, being an important question that of the compactness of such representations, we are not aware of many results in this subject. This is the main gap we have tried to fill in this work: What is the minimum number of lines needed to represent (either to p-embed - without crossing of edges - or to grid locate allowing edges crossing-) in the grid a given graph?

Regarding p-embeddability, although it is known that any planar graph can be p-embedded in the plane, the question of the minimal area needed to obtain such representation is still open, since we have provided only partial results concerning bipartite graphs.

On the other hand, regarding grid location, we have proved that a 4-regular graph (non-isomorphic to $K_{5}$ ) can be located in three lines, and that if a graph is not regular and its maximal degree is 4 , then it can be located in two lines. Other cases are still open.

\section{References}

1. Balko, M.: Grid Representations and the Chromatic Number. In: 28th European Workshop on Computational Geometry, pp. 45-48 (2012)

2. Barrière, L., Huemer, C.: 4-Labelings and Grid Embeddings of Plane Quadrangulations. In: Eppstein, D., Gansner, E.R. (eds.) GD 2009. LNCS, vol. 5849, pp. 413-414. Springer, Heidelberg (2010) 
3. Brooks, R.L.: On colouring the nodes of a network. Cambridge Philosophical Society, Math. Phys. Sci. 37, 194-197 (1941)

4. Chrobak, M., Nakano, S.: Minimum width grid drawings of plane graphs. Computational Geometry 11(1), 29-54 (1998)

5. Cornelsen, S., Schank, T., Wagner, D.: Drawing Graphs on Two and Three Lines. In: Goodrich, M.T., Kobourov, S.G. (eds.) GD 2002. LNCS, vol. 2528, pp. 31-41. Springer, Heidelberg (2002)

6. Dolve, D., Leighton, F.T., Trickey, H.: Planar embedding of planar graphs. Advances in Computing Research. VLSI Theory, vol. 2. JAI Press, Inc., Greenwich (1984)

7. Fraysseix, H., Pach, J., Pollack, R.: How to draw a planar graph on a grid. Combinatorica 10(1), 41-51 (1990)

8. Flores-Peñaloza, D., Santos, F., Zaragoza, F.J.: Encajes primitivos de gráficas aplanables. In: XXVII Coloquio Víctor Neumann (2012)

9. Flores-Peñaloza, D., Zaragoza, F.J.: Every four-colorable graph is isomorphic to a subgraph of the visibility graph of the integer lattice. In: 21st Canadian Conference on Computational Geometry, CCCG 2009, pp. 91-94 (2009)

10. Hardy, G.H., Wright, E.M.: An introduction to the theory of numbers. Oxford University Press (1979)

11. Kára, J., Pór, A., Wood, D.R.: On the chromatic number of the visibility graph of a set of points in the plane. Discrete and Computational Geometry 34(3), 497-506 (2005)

12. Por, A., Wood, D.R.: On visibility and blockers. J. Computational Geometry 1(1), 29-40 (2010) 